\title{
Optimization of stacker control system based on laser ranging and bar code localizing technology
}

\author{
Peng Zhang ${ }^{\mathrm{a}}$, Teng Zhang and Daixi Li \\ School of Mechanical and Automotive Engineering, Qilu University of Technology, 250353 Jinan, China
}

\begin{abstract}
The target goods location of some stackers is realized by the recognition site piece. The point-to-point communication mode is used, such as infrared communication, leading to low localizing precision, reliability and poor scalability. To overcome the above shortcomings, taking a logistic laboratory's stacker of university for example, the original control system is improved by laser ranging, barcode localizing technology and industrial wireless Ethernet technology. Results show that the running reliability and repeat localizing accuracy of the stacker is improved obviously.
\end{abstract}

Keywords: wireless ethernet; laser ranging; bar code positioning; stacker.

\section{Introduction}

Recent years, with the rapid development of automatic storage system, stacker is the core equipment. But in some enterprises, traditional recognition site localizing mode is combined with encoder that has been adopted as the main method by the stacker, which is low precision and can lead to errors through each access of goods. Then the efficiency has never been able to be improved. With the emergence of laser technology, which makes the ranging method constantly updated, as well as measurement accuracy has been greatly improved. The following is the laser as the light source of the optical ranging finder to replace the traditional ranging method. At present, automated storage enterprises in foreign countries adopt the laser ranging and positioning technology, and have been studying the new positioning technology, such as acoustic positioning technology. In China, the communication of the stacker between automatic storage system and other devices is mainly using infrared and fieldbus mode. Although this method has been mature, the following problems are sure: transmission distance is limited; low reliability, because it is easy to be blocked and cause communication interruption; it is poor by using point-to-point communication and scalability, not suitable for curved roadway, like circular roadway. Take logistic laboratory in a university for example, laser ranging, barcode localizing technology and industrial wireless Ethernet are used to improve the stacker control system, to achieve high-precision localizing and increase the automation level [1-3].

a Corresponding author : sqzhangpeng@163.com

(C) 2016. The authors - Published by Atlantis Press 


\section{Control system of stacker}

\subsection{Control system architecture of stacker}

The recognition site of logistic laboratory storage system in a university is used by the original stacker to accomplish goods grid location, traditional wired way is used to ensure data communication of all devices. On the basis of the original equipment, its control system is optimized and improved, and recognition site is replaced by barcode scanner and laser range finder, to improve the repeated localizing precision. Wireless Ethernet communication is used in automatic storage system to overcome the shortcomings of traditional method of communication between the stacker controller and the monitor computer [4-6]. The control system network structure of stacker is shown in Figure 1.

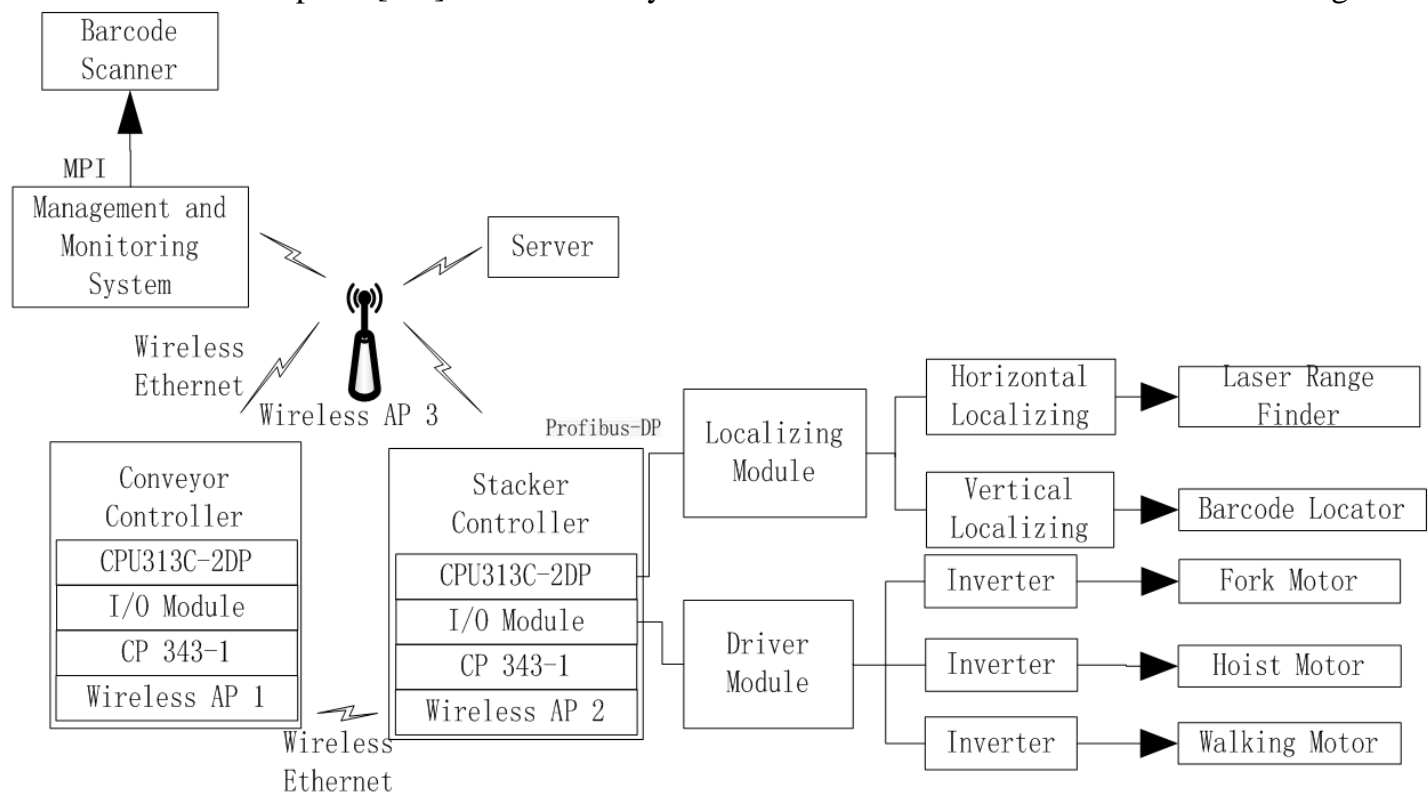

Figure 1. Control system network structure of stacker

Control system network structure of stacker contains four aspects:

(1) MPI interface is used to connect management and monitoring systems and barcode scanners, which the data communications follow MPI transfer protocol. When there are goods in/out warehouse, the bar code scanner begins to scan barcode tape which is attached on cargo box, then read information to the management and monitoring system. Finally, the mission is reached to the stacker, and goods automated in/out are accomplished by the stacker.

(2) Data transmission between the server and the management and monitoring system follows Ethernet transfer protocol, which ensures transmission of data through the wireless AP. Server is used to provide data information to be collected when storage system running, like location of goods, order date. The management of operator and the real-time monitoring of the running state of the conveyor and the stacker are both realized by management and monitoring system.

(3) Two wireless AP modules are used to realize data wireless transmission of conveyor controller and stacker controller, which follows Ethernet transfer protocol too. Digital input port of the MM440 inverter is used to connect driving module with the controller of stacker, to ensure corresponding actions according to the task.

(4) The laser range finder and the barcode scanner of the localizing module are connected with the PLC controller of stacker, which follow Profibus-DP transfer protocol [7]. The horizontal localizing of the grid is realized by laser range finder. Bar code localizing system is used to ensure the vertical localizing of the g rid. 


\subsection{Grid horizontal localizing system}

The grid horizontal localizing of logistic laboratory stacker is using DL100 series laser range finder of Sick Company in Germany. It emits laser to a certain distance between the reflector, according to the change in the distance between the laser range finder and the reflector, to process this change signal and transmit to the PLC memory module, thus control of the stacker. When the stacker moving, as its unique PLC synchronous technology, the distance value can be updated in real time, and the running process of stacker in low, medium and high speed for feedback can be controlled in real time, to ensure the localizing precision and the stability of stacker [8-9].

The laser range finder is fixed on the stacker, and the reflector is fixed on the other end of the rail. It should be noted, to improve major shortcomings of previous recognition site error, when the stacker after running for a long time, laser range finder will produce small offset because of stacker running vibration, and reflector may absorb dust more or less, this phenomenon will produce effect on the localizing precision. As a result, laser range finder adjusted periodically and reflector cleaned regularly must be done by operator, to ensure normal operation of stacker. Laser range finder application in the laboratory is shown in Figure 2.

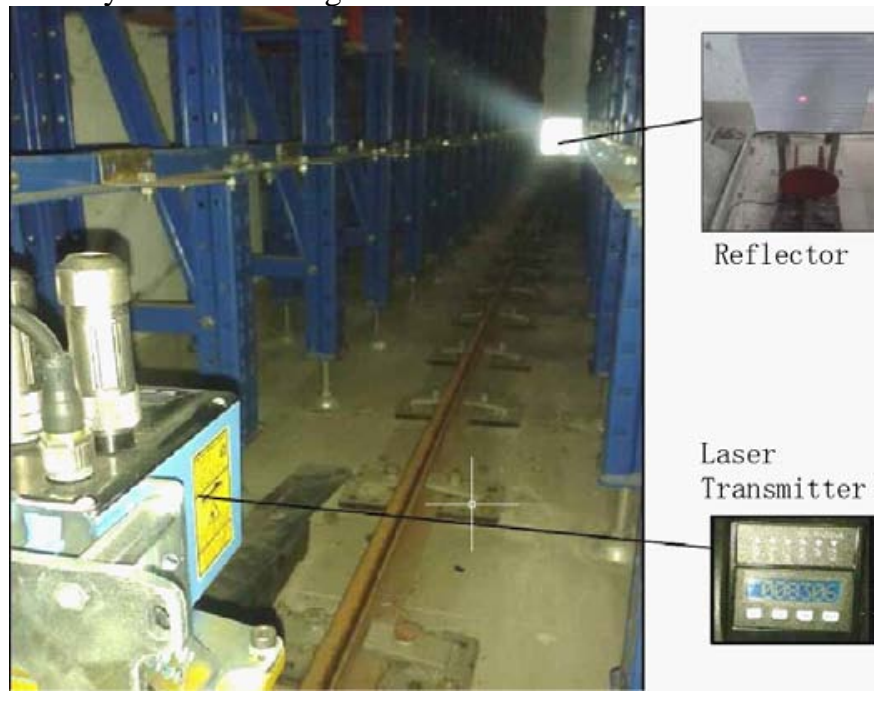

Figure 2. Laser range finder in the laboratory

Because of laser range finder for micron level precision, even stacker in the absence of work state, the number is still changing, which displayed on the laser range finder. To ensure that the read number of laser range finder and the address of internal memory storage goods shelf can be compared with each other, the precision of laser range finder is made down to millimetre level by programming and designing.

\subsection{Grid vertical localizing system}

The grid vertical localizing of logistic stacker is using BPS348iSM100D barcode localizing instrument of Leuze electronic Company in Germany. The BPS barcode localizing instrument includes barcode tape and barcode reader that are fixed on the side of an upright post of a stacker and one side of the loading platform, respectively. During goods ascending and declining, barcode reader scanning four tapes at the same time, takes the contained data information to determine the current position. For calculating the accurate distance of the loading platform ascension and decline, the information is uploaded to the PLC controller for stacker processing[10]. In the laboratory the stacker barcode localizing instrument is shown in Figure 3. 


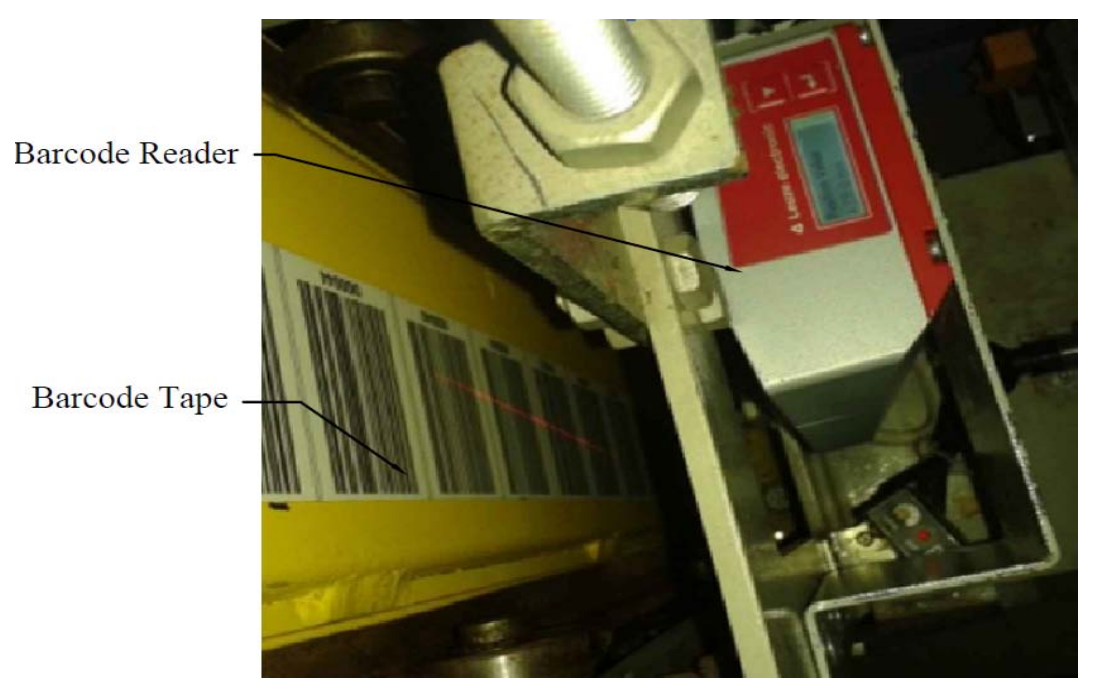

Figure 3. Barcode localizing instrument

Barcode localizing system can achieve repeatability precision of $\pm 1 \mathrm{~mm}$ millimeters. Because of the barcode locator installed for a certain reading distance, even if the equipment vibration caused by trace deviation, it can guarantee the precision of localizing.

\subsection{Moving control of stacker}

Two electromagnetic braking motors and an ordinary three-phase AC motor are drived by three MM440 Siemens inverters[11]. Two electromagnetic braking motors are used to drive stacker moving and loading platform ascending and declining. Fork moving of stacker is drived by three-phase AC motor. The inverter is used to realize smooth switching control of stacker in high speed, medium speed and low speed. The speed characteristic curve of stacker is shown in Figure 4.

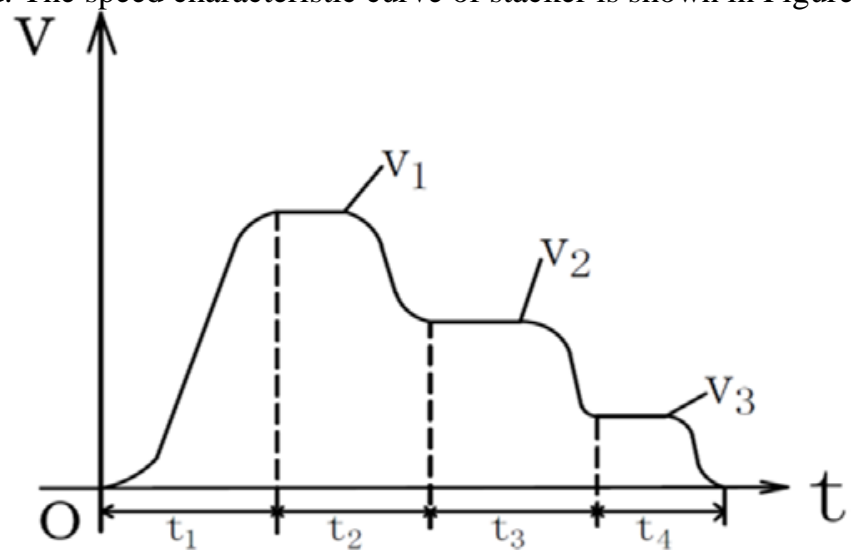

Figure 4. Speed characteristic curve of stacker

Stacker running speed curve diagram is divided into four slots: $\mathrm{t} 1$ is the time for the stacker after accepting its task, to smoothly accelerate from the velocity of zero to that of high speed V1; 22 is the time that the stacker just reaches to the high speed V1, and decelerates to the medium speed V2 smoothly when the distance of the stacker being away from the target position is two grids; $\mathrm{t} 3$ is the time that the stacker just reaches to the medium speed V2, and smoothly decelerates to the low speed V3 when the stacker just reaches to the target frame; $\mathrm{t} 4$ is the time that the stacker stop accurately from the velocity of low speed V3 to that of zero. The experiment result shows that the stacker can be 
smoothly switched between three kinds of speed, to avoid the impact to the effects of motor speed, and extend the life of the motor.

\section{PLC control program of stacker}

\subsection{Control program structure of stacker}

The main control program of stacker includes system control, stacker control, task processing, goods address information, and precision conversion of the laser range finder program module. The system control program is programming mainly for three real-time displays of the stacker operation, fault, and warning states. Stacker control program is used to control stacker according to the task. Task handle program which is used to guarantee stacker accepts distributed task from management and monitoring system, and the task processing result is returned to the management and monitoring system. Grid's address information program is mainly used to sort each address information in storage system, and address information is stored in the storage for PLC controller to read. Laser ranging precision conversion program is used to ensure normal use of the laser ranged finder. With the program design, the actions of the stacker are transformed into logic signals for processing, to satisfy the control requirement of stacker. Control program structure is shown in Figure 5.

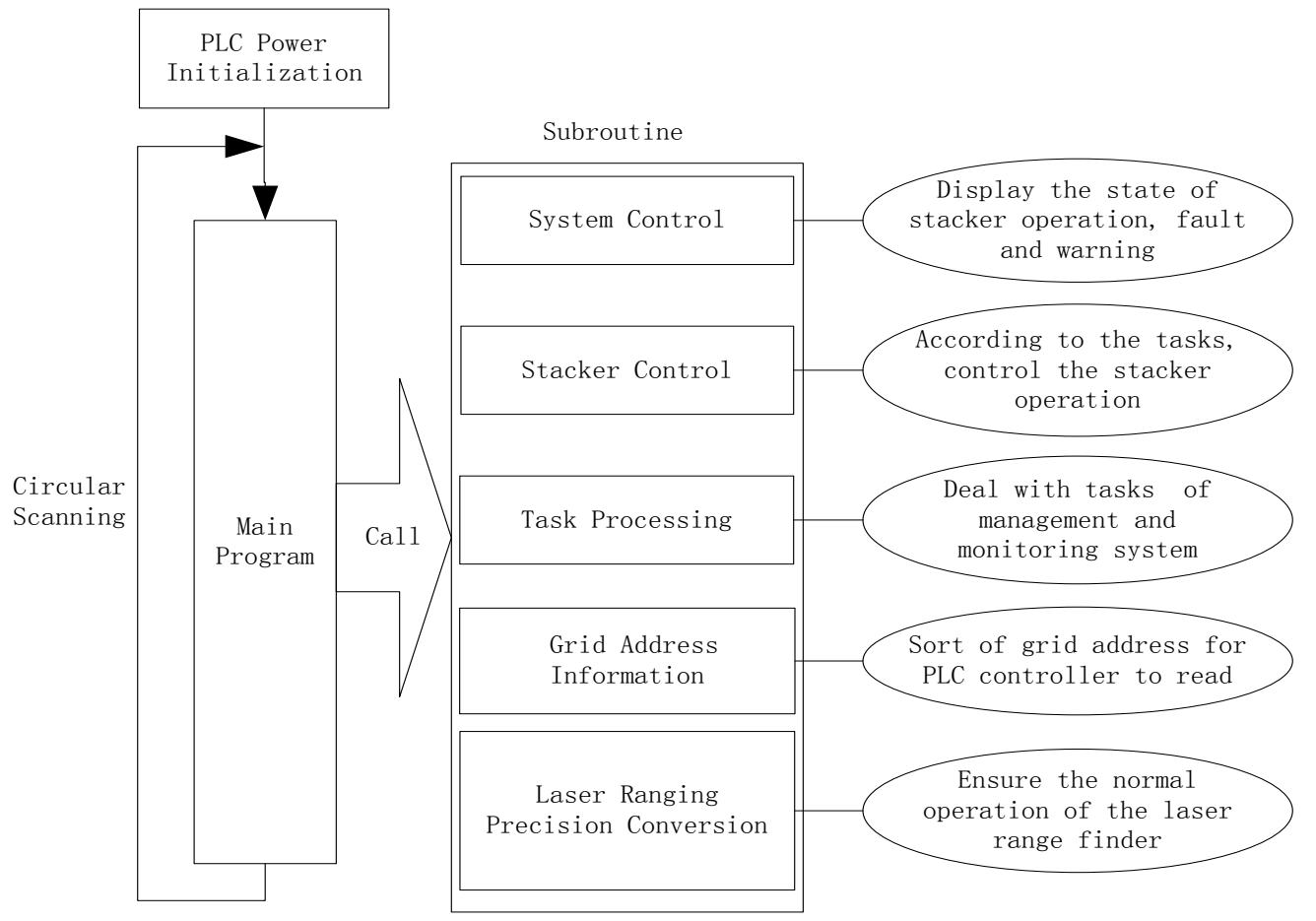

Figure 5. Control program structure of stacker

\subsection{Data communication protocol of stacker running states}

Stacker is running according to the task, and running states must be returned to the management and monitoring system, to ensure master the operation of stacker in real-time. Stacker starts to accept the messages of the task, forward and backward, fork's movement, loading platform's ascension and decline, which are reflect the running state of the stacker. To make each running state of stacker can be returned to the management and monitoring system in time, the operation of the stacker shall 
comply with the necessary data communication protocol. Part of the stacker operation states data exchange protocols are shown in table 1.

Table 1. Data exchange protocols

\begin{tabular}{|c|l|l|}
\hline Data dress & \multicolumn{1}{|c|}{ Name } & \multicolumn{1}{c|}{ Meaning of protocol code } \\
\hline DB12.DBB 7 & $\begin{array}{l}\text { Stacker state } \\
\text { display }\end{array}$ & $\begin{array}{l}\text { 0:no mission, 1:start,2: task received, 3:pickup completed, } \\
\text { 4:task completed, 5:right fork,6;leftfork,7:ascend, 8:decline, } \\
\text { 9:forward, 10:backward }\end{array}$ \\
\hline DB12.DBB 8 & $\begin{array}{l}\text { Stacker fault } \\
\text { display }\end{array}$ & $\begin{array}{l}\text { 0: normal, 1: inverter fault, 2: inventory conflict, } \\
\text { 3: pickup conflict, 4: fork timeout, 7: goods on the outlet }\end{array}$ \\
\hline DB12.DBB 9 & $\begin{array}{l}\text { Accept new } \\
\text { task or not }\end{array}$ & 0:allow, 1:ban \\
\hline DB12.DBB10 & $\begin{array}{l}\text { Stacker } \\
\text { running mode }\end{array}$ & 0:manual, 1:automatic \\
\hline
\end{tabular}

\section{Repeated localizing precision after optimizing}

Traditional recognition site is used by the stacker in the storage logistic laboratory to realize goods location before the system optimization. There are shortcomings like a fork moving cannot reach to designated position, goods easy to fall during operation processing, and grid repeated localizing precision is $\pm 10 \mathrm{~mm}$. By optimizing the allocation of software and hardware of the control system, grid horizontal repeated localizing precision can reach to $\pm 0.5 \mathrm{~mm}$, and vertical repeated localizing precision can reach to $\pm 1 \mathrm{~mm}$.

\section{Conclusion}

In this paper, a logistic laboratory of university taken for example, control system optimization of stacker and implementation method of storage logistic laboratory in detail is discussed. After the control system optimization of stacker, the running speed characteristics of stacker are made by an intuitive graphic. The experiment indicates that the stacker runs stably, and grid localizing precision is further improved. It provides the reference to the control system upgrade of stacker.

\section{Acknowledgements}

This paper is supported by the independent innovation university project of Ji'nan (201202079).

\section{References}

1. Cheng Bin, Jin Guofeng. Address technology research of stacker. Journal of logistics technology and application, 100-101, 6, (2010)

2. Xu Ling, Lao Yangjian. Control system design of stacker based on PLC. Journal of combination machine tools and automatic processing technology, 72-74, 1, (2005)

3. Yao Jianhui, Yu Chao, Wang Wenming. Binning stacker research design. Journal of agricultural mechanization research, 89-91, 9, (2010)

4. Shen Zhi. Automated control system design of stacker based on S7-200 PLC. Industrial automation, 130-133, 3, (2010)

5. Wang Lingqi. Automated warehouse location address detection and monitoring. Journal of combination machine tools and automatic processing technology, 18-20, 9, (2002)

6. Duan Zhimin, Chen Jingjie. An economical stacker and control system. Journal of mechanical design and manufacturing, 46-48,2,(2002)

7. Du Zhiyong, Wang Xianfang. Medium-sized warehouse with PLC to realize control. Journal of 
laboratory research and exploration,34-37,11,(2004)

8. Leng Jianwei, LI Tao. Bridge crane localizing based on laser ranging system design. Electric drive, 48-50,7,(2011)

9. Han Jun, Lu Zonghui. Control ways of stacker localizing. Journal of manufacturing automation, $1-2,37,(2015)$

10. Cheng Weifang, Peng Yu.Workpiece-in-process management based on barcode technique. China Mechanical Engineering,1384-1388,13,(2006)

11. Lu Jianguo. Application of MM440 inverter in variable pump testing system. Research and Exploration in Laboratory,185-187,4,(2011) 